\title{
A NOTE ON KANTOROVICH TYPE BERNSTEIN CHLODOVSKY OPERATORS WHICH PRESERVE EXPONENTIAL FUNCTION
}

\author{
Ali Aral, Didem Aydin ARI AND BAŞAR YILMAZ
}

\begin{abstract}
This paper is mainly focused on the integral extension of Bernstein-Chlodovsky operators which preserve exponential function. Inspire of the Bernstein-Chlodovsky operators which preserve exponential function, we define the integral extension of these operators by using a different technique. We give weighted approximation properties including a weighted uniform convergence and a weighted quantitative theorem in terms of exponential weighted modulus of continuity. Furthermore, we give the Voronovskaya type theorem.
\end{abstract}

Mathematics subject classification (2020): 41A25, 41A36.

Keywords and phrases: Voronovskaja type theorem, weighted modulus of continuity, rate of convergence.

\section{REFERENCES}

[1] I. Chlodowsky, Sur le développement des fonctions définies dans un intervalle infini en séries de polynomes de M. S. Bernstein, Compositio Math., 4 (1937), 380-393.

[2] L. V. Kantorovich, Sur certains d'eveloppementssuivant les polynmes de la forme de S. Bernstein, I, II, C. R. Acad. URSS; 563-568 and 595-600, 1930.

[3] F. Altomare And M. Campiti, Korovkin-type Approximation Theory and Its Applications, de Gruyter (New York, 1994).

[4] A. D. GADJIEV, The convergence problem for a sequence of positive linear operators on bounded sets and theorems analogous to that of P. P. Korovkin, Dokl. Akad. Nauk SSSR 218 (5) (1974); Transl. in soviet Math. Dokl. 15 (5) (1974) 1433-1436.

[5] A. D. GadjIEv, On P. P. Korovkin type theorems, Math. Zametki 20 (1976) 781-786; Transl. in Math. Notes (5-6) (1978) 995-998.

[6] R. PĂLTăNEA, A note on Bernstein-Kantorovich operators, Bull. Univ. Transilvania of Braşov, Series III 6 (55) (2), 27-32 (2013).

[7] A. Aral, D. Otrocol \& I. RaşA, On a New Type of Bernstein-Kantorovich Operators, Period Math Hung (2019), https://doi.org/10.1007/s10998-019-00284-3.

[8] T. Acar, A. Aral, D. Cárdenas-Morales, P. Garrancho, Szász-Mirakyan Type Operators Which Fix Exponentials, Results in Mathematics 72 (2) 2017, 1393-1404.

[9] A. HolHos, The Rate of Approximation of functions in an infinite interval by positive linear operators, Stud. Univ. Babes-Bolyai Math. 2, 133-142 (2010).

[10] V. Gupta AND A. ARAL, A note on Szász-Mirakyan-Kantorovich type operators preserving $e^{-x}$, Positivity (2018) 22: 415-423.

[11] A. Aral, D. Cárdenas-Morales, P. Garrancho, I. Raşa, Bernstein-type operators which preserve polynomials, Computers and Mathematics with Applications 62 (2011) 158-163.

[12] F. ÖZsarac, A. Aral and H. Karsli, On Bernstein-Chlodovsky Type Operators Preserving Exponential Functions, ICRAPAM 2018. Springer Proceedings in Mathematics \& Statistics, Vol. 306, Springer, Singapore.

[13] M. Bodur, Ö. Gürel Yilmaz, A. Aral, Approximation by Baskakov-Szász-Stancu Operators Preserving Exponential Functions, Constructive Mathematical Analysis, 2018; 1 (1): 1-8.

[14] T. ACar, M. Murs aleen, S. N. Deveci, Gamma operators reproducing exponential functions, Adv. Difference Equ., 2020: 423. 
[15] T. Acar, A. Aral, S. A. Mohiuddine, On Kantorovich modification of $(p, q)$-Bernstein operators, Iran. J. Sci. Technol. Trans. A Sci. 42 (2018), no. 3, 1459-1464.

[16] V. Gupta, G. TACHeV, A. M. ACU, Modified Kantorovich operators with better approximation properties, Numer. Algorithms 81 (2019), no. 1, 125-149.

[17] V. Tотік, Problems and solutions concerning Kantorovich operators, J. Approx. Theory 47, (1983), 51-68.

[18] P. N. Agrawal, M. Goyal, Generalized Baskakov Kantorovich Operators, Filomat, 31:19 (2017), 6131-6151.

[19] H. H. GonsKa AND X.-L. Zhou, The Strong Converse Inequality for Bernstein-Kantorovich Operators, Computers Math. Applic. Vol. 30, No. 3-6 (1995), 103-128. 